\title{
Immunoexpression of CD30 and CD30 ligand in deciduas from spontaneous abortions
}

\author{
M. Trovato, ${ }^{1}$ M. Grosso, ${ }^{1}$ E. Vitarelli, ${ }^{1}$ M. Le Donne, ${ }^{2}$ V. Barresi, ${ }^{1}$ F. Trimarchi, ${ }^{3}$ G. Barresi ${ }^{1}$ \\ ${ }^{1}$ Dipartimento di Patologia Umana, Padiglione D, Policlinico Universitario, G. Martino, Messina; \\ 2Dipartimento di Scienze Ginecologiche, Ostetriche e Medicina della Riproduzione, Policlinico \\ Universitario, G. Martino; ${ }^{3}$ Sezione di Endocrinologia, Dipartimento Clinico-Sperimentale di Medicina e \\ Farmacologia, Policlinico Universitario, G. Martino, Messina, Italy
}

i.

(C)2005, European Journal of Histochemistry

In the present study, using immunohistochemistry, we studied the expression of CD30 and CD30-L in 35 deciduas obtained from women following elective abortion during normal physiological gestation and in 60 deciduas obtained from women after spontaneous abortion with or without signs of inflammation. The main difference was noticed in the first trimester of gestation in which was found a decrease in CD30/CD30-L-positive decidual glandular and stromal cells in a greater number of cases of spontaneous abortions with respect to cases of physiological pregnancies (70\% vs $50 \%, p<0.05)$. In addition, deciduas from spontaneous abortions with inflammation and without inflammation reacted similarly. The reduced expression of CD30 and CD30-L and their cellular pattern detected in the deciduas from spontaneous abortions suggest that the CD30/CD30-L system is crucial for preventing abortions in the first trimester. And furthermore, the distinctive expression of CD30/CD30$L$ in deciduas from physiological pregnancies may indicate that the CD30/CD30-L system exerts its main role in the first trimester.

Key words: CD30; CD30 ligand; Human Deciduas; Abortions; Immunohistochemistry.

Correspondence: Maria Trovato,

Dipartimento di Patologia Umana, Padiglione D, Policlinico

Universitario G. Martino, 98125 Messina, Italy

Tel: +39.090 .2212543 .

Fax: +39.090.2212523.

E-mail:mariatrovato@tin.it

Paper accepted on May 16, 2005

European Journal of Histochemistry

2005; vol. 49 issue 3 (Jul-Sep): 285-290
$\mathrm{P}$ regnancy failures are largely attributed to the excessive production of Th-1 cytokines in cases where genetic, anatomic, endocrine and infectious factors can be ruled out (Raghupathy et al., 1999; Salomon, 2004). On the contrary, Th-2 and Th-3 cytokines favour the maintenance of pregnancies (Clark and Croitoru, 2001). Several studies have proposed that tumor necrosis factor (TNF) superfamily members contribute to a successful pregnancy by regulating the Th- $1 /$ Th- $2 /$ Th- 3 balance with a bias toward Th-2 cytokines at the maternal-fetal interface (Locksley et al., 2001; Phillips et al., 2003). Moreover, recent studies indicate that the ligation of CD40, a member of the TNF receptor superfamily, causes pregnancy failure by acting on the reproductive endocrine system via inhibition of the hypothalamic-pituitary-gonadal axis, even in the absence of an inflammatory injury in decidual tissue (Erlebacher et al., 2004). Consistent with these data, CD40 is reported not to be expressed at the maternal-fetal interface in the human placenta (Phillips et al., 2003).

To better clarify the role of TNF receptors in human physiological gestation, and their potential clinical implications for preventing abortions, we studied CD30, a member of TNF superfamily receptor, and its cognate ligand, CD30-L. CD30 is a cytokine receptor showing an extracellular domain composed of 365 residues containing several cysteine-rich pseudorepeats, cross-linking the membrane-bound cytokine, namely, CD30-L (Durkop et al., 1992; Smith et al., 1993; Falini et al., 1995). CD30 and CD30-L are characterized as specific markers of the Reed-Sternberg cells in Hodgkin's disease, and both are found in normal and malignant lymphatic cells, activated B and T lymphocytes and in Burkitt lymphoma cells (Schwab et al., 1982; Gruss et al., 1996; Burns and Dardick, 1990; Gruss et al., 1996). Furthermore, malignant mesenchymal and epithelial cells are recognized as being able to express CD30 and CD30-L 
(Mechtersheimer and Moeller, 1990; Trovato et al., 2001; Ruggeri et al., 2002). Depending on the cell type, different effects have been attributed to the CD30/CD30-L interaction, such as the enhancing of cellular growth or apoptosis (Gruss et al., 1994; Lee et al., 1996). Specifically, it has been shown that CD30 could alter the levels of Th-2 and Th-1 cytokines, triggering mechanisms involved in the regulation of the balance between Th-2/Th-1 functions (Pellegrini et al., 2003).

The presence of $C D 30$ and $C D 30-L$ in first trimester and term placentas has been amply documented, thus suggesting their possible regulatory role in this tissue (Ito et al., 1994; Durkop et al., 2000; Sverremark-Ekström et al., 2001; Papadopoulus et al., 2001; Phillips et al., 2003). CD30 has been described in decidual cells (Ito et al., 1994; Durkop et al., 2000; SverremarkEkström et al., 2001; Papadopoulus et al., 2001), whereas CD30- $L$ expression has been recognized in Hofbauer cells as well as in the decidua and in endothelial cells (Sverremark-Ekström et al., 2001; Phillips et al., 2003).

The aim of the present study was to investigate the immunoexpression of both CD30 and CD30-L in deciduas from physiological pregnancies and in deciduas from spontaneous abortions, either with or without inflammatory cell infiltration, during the three gestational trimesters.

\section{Materials and Methods}

\section{Tissue Collection}

The investigations were approved by the Ethics Committee of the medical faculty of Messina University. A total of 95 deciduas were retrieved from the files of the Department of Human Pathology, University of Messina. Sixty were first trimester deciduas (ranging from 7-12 weeks). Of these, 20 were obtained from voluntary abortions of physiological pregnancy (VA), 20 from spontaneous abortions associated with features of decidual inflammation on the basis of histological observations without a specific infective etiology (SADI), and 20 from spontaneous abortions without decidual inflammation (SA-WDI).

Twenty-five were second trimester deciduas (ranging from 13-24 weeks) pertaining to 5 VA, 10 SA-DI and 10 SA-WDI cases.

The final 10 were third trimester deciduas (ranging from 36-42 weeks), taken from normal sponta- neous vaginal deliveries.

After each delivery, samples of placenta including decidual floor were immediately fixed in $4 \%$ formalin. The samples were then processed routinely through graded alcohol and xylene to paraffin wax. Haematoxylin-eosin stained sections of each specimen were made. Each decidua was isolated from the co-respective placental specimen showing no histological or microscopic features of chorionamnionitis.

\section{Immunohistochemistry}

Serial sections of the selected blocks were cut at $5 \mu \mathrm{m}$ for the immunohistochemical studies. Immunohistochemistry was performed using mouse monoclonal antibodies raised against human CD30 (or Ki-1 antigen) (clone Ber-H2, Dako, Carpinteria, CA, USA) and human CD30-L (h-CD30L-Fc type II, Genzyme, Cambridge, MA, USA), respectively. The biotin-streptavidin-peroxidase method (LSAB kit from Dako Corporation, Carpinteria, CA) was employed to reveal the immunoreaction. We used the antigen retrieval technique as described by Gown et al., (1993). Slides were deparaffinized in xylene, rehydrated and then the endogenous peroxidase activity was quenched by adding $3 \%$ hydrogen peroxide for $15 \mathrm{~min}$. Sections were microwaved for 15 min (Whirlpool AVM 300, power set at 500 watts). Microwave exposure was broken into three equal time periods and, at the end of the first cycle, $50 \mathrm{ml}$ of distilled water was added to the slide holder to prevent loss of fluid from boiling. The reaction was developed by 3,3'-diaminobenzidine (DAB) with $0.05 \%$ hydrogen peroxide as chromogen. Sections were counterstained with Mayer's haematoxylin, dehydrated and mounted. Specificity was assessed by omitting the primary antiserum or replacing the primary antiserum with normal mouse serum. In addition, an immunoabsorption test was performed to confirm the specific immunoreactivity of each antibody. As positive controls for CD30-L and CD30, tissue slides taken from specimens of Hodgkin's lymphoma were tested. For the evaluation and comparison of the results using CD30 and CD30-L antibodies, the following criteria were used: (1) number of positive cases; (2) number of positive decidual glandular and stromal cells per case; the count of the number of the reactive cells was based on evaluation of 500 cells for each case using a $50 \mathrm{X}$ magnification; (3) site of reaction: cytoplasm. Immunohistochemical evalua- 
Table 1. Expression of CD30 and CD30-L in deciduas of physiological pregnancies and spontaneous abortions. PhPa, deciduas of physiological pregnancies; SA-Ib, deciduas of spontaneous abortions with inflammation signs; SA-WIc, deciduas of spontaneous abortions without inflammation signs.

\begin{tabular}{|c|c|c|c|c|c|c|}
\hline & $I^{\circ}$ trimester & & $I^{\circ}$ trimester & & $111^{\circ}$ trimes & \\
\hline & CD30 & CD30-L & CD30 & CD30-L & CD30 & CD30-L \\
\hline PhPa & $14 / 20$ & $14 / 20$ & $2 / 5$ & $2 / 5$ & $0 / 10$ & $2 / 10$ \\
\hline$S A-I^{b}$ & $10 / 20$ & $10 / 20$ & $2 / 10$ & $2 / 10$ & & \\
\hline$S A-W I^{c}$ & $10 / 20$ & $10 / 20$ & $2 / 10$ & $2 / 10$ & & \\
\hline
\end{tabular}

tions of all deciduas were performed twice and blindly by different pathologists (M.T., M. G., G. B.) with an inter-observer concordance of nearly $100 \%$. When discrepancy was present, the mean value was considered.

\section{Statistical Analysis}

Immunohistochemical results were expressed as mean \pm S.D. Statistical analysis was performed with the Primer statistical program. Differences between means were assessed by the non-parametric test of Mann-Whitney and differences between proportions with the $\chi^{2}$ test with Yates correction for continuity. Significance was set at $5 \%$.

\section{Results}

The immunohistochemical data are summarized in Tables 1-4. In all positive deciduas, the immunostaining for both CD30 and CD30-L was localized in the cytoplasm of glandular and stromal cells (Figure 1). No CD30 or CD30-L immunostaining was detected in lymphocytes. In deciduas from physiological pregnancies, the immunoexpression for CD30 and CD30-L was encountered in 14 of the 20 cases $(70 \%)$ in the first trimester of gestation, and in 2 of the 5 cases $(40 \%)$ in the second trimester. In the third trimester, only the CD30-L immunoreaction was noticed in 2 of the 10 cases $(20 \%)$ (Table 1). Moreover, in deciduas from the physiological pregnancies of the first trimester of gestation, the percentage of glandular cell reactivi- ty for both CD30 and CD30-L was higher than stromal cell reactivity, with a significantly higher expression of CD30-L $(p<0.05$, respectively) (Table 2$)$. Furthermore, in these deciduas, a decrease in glandular and stromal cell positivity for both CD30 and CD30- $L$ was observed in the second and third trimester of gestation ( $p<0.05$, respectively) (Table 2 ). The immunoreaction for CD30 and CD30-L in deciduas from spontaneous abortions either with or without inflammation signs was positive in 10/20 cases $(50 \%)$ in the first trimester of gestation and in $2 / 10$ cases $(20 \%)$ in the second trimester of gestation (Table 1). Moreover, in the first trimester of gestation, deciduas from spontaneous abortion with or without inflammatory signs expressed CD30 and CD30- $L$ in a significantly reduced number of glandular and stromal cells in comparison to those of the physiological pregnancy deciduas $(p<0.05$, respectively), with the exception of stromal cell CD30 immunoreactivity in the deciduas with inflammation ( $p=0.10$ ) (Tables 2-4).

\section{Discussion}

Cytokines play a major role in the success of physiological gestation by acting on multiple pathways and activating different signals according to the source of their production and period of gestation. The cytokines and growth factors produced by endometrial cells through estradiol and progesterone stimulation seem to be responsible for the extensive remodeling of the endometrium during

Table 2. Expression of CD30 and CD30-L in glandular and stromal cells of physiological pregnancies deciduas.

\begin{tabular}{|c|c|c|c|c|c|c|}
\hline & $I^{\circ}$ trimester & & $\|^{\circ}$ trimester & & III ${ }^{\circ}$ trimeste & \\
\hline & CD30 & CD30-L & CD30 & CD30-L & CD30 & CD30-L \\
\hline Glandular cells & $* 17.8( \pm 5.4)$ & $29.2( \pm 5.1)$ & $7.5( \pm 3.5)$ & $10( \pm 5)$ & $0( \pm 0)$ & $3( \pm 1.4)$ \\
\hline Stromal cells & $13.5( \pm 4.5)$ & $22.8( \pm 6.7)$ & $3.5( \pm 0.7)$ & $5( \pm 2)$ & $0( \pm 0)$ & $2.5( \pm 0.7)$ \\
\hline
\end{tabular}


Table 3. Expression of CD30 and CD30-L in glandular and stromal cells of spontaneous abortion deciduas with inflammation signs.

\begin{tabular}{|c|c|c|c|c|}
\hline & $I^{\circ}$ trimester & & $I^{\circ}$ trimester & \\
\hline & CD30 & CD3O-L & CD30 & CD3O-L \\
\hline Glandular cells & $* 10.5( \pm 3.6)$ & $12.5( \pm 2.6)$ & $4( \pm 1.4)$ & $8.7( \pm 2.5)$ \\
\hline Stromal cells & $10( \pm 3.3)$ & $12( \pm 2.5)$ & $2.5( \pm 0.7)$ & $6.2( \pm 2.5)$ \\
\hline
\end{tabular}

*mean ( $\pm S D$ )

blastocyst implantation and placenta formation; involving, initially, the secretory transformation of the glandular epithelium, followed by edema and decidualization of the stromal compartment (Lockwood et al., 1993; Ghosh and Sengupta, 1998; Fazleabas and Strakova, 2002). The cytokines, chemokines and prostaglandins, secreted from the immunocompetent cells present in deciduas, such us macrophages, natural killer cells and T cells, are involved in the regulation of the immunoresponse at the maternal-fetal interface with the consequent control of trophoblast survival and death by balance of growth/apoptosis stimulation (Saito, 2001; Wegmann et al., 1993; Carson et al., 2000). The cytokines produced throughout gestation by systemic immunocompetent cells are able to regulate the expression of genes driving progesterone synthesis in the reproductive endocrine system (Erlebacher et al., 2004). The TNF ligand/receptor system has been indicated as a good model to study possible targets for therapeutic strategies aimed at preventing recurrent early pregnancy loss (Salmon, 2004). In the present study, we have investigated the immunoexpression of CD30 and CD30-L in deciduas from physiological pregnancies and from spontaneous abortions. In deciduas from physiological pregnancies, a marked reduction in CD30 and CD30-L immunoreactivity was detected in the second and third trimester in comparison to the first trimester of gestation. Therefore, it appears that the role played by the CD30/CD30-L system in physiological pregnancies is specific and restricted to the first trimester.

Both CD30 and CD30-L were detected in the glandular and stromal cells, while no CD30 or CD30-L immunostaining was observed in the immunocompetent cells of the reactive deciduas. These data permit us to retain that the CD30/CD30-L system may have a role in the transformation of the endometrial mucosa in deciduas. Lastly, considering that the glandular cells of physiological gestation deciduas exhibited the highest expression of CD30 and CD30-L, it may be assumed that glandular cells showing CD30 and CD30- $L$ are among the decidual target cells favoring the efficient implantation of placenta on the maternal tissue.

In this study, we have also identified, in the first trimester of gestation, a significantly reduced reactivity for CD30 and CD30-L in the deciduas of spontaneous abortions with respect to those of physiological pregnancies. These data emphasize that the role played by the CD30/CD30-L system in the period restricted to immediate postimplantation, is crucial for preventing abortions in the first trimester. Furthermore, the highest expression of the ligand with respect to the receptor in the first trimester deciduas from physiological gestations and the similar expression of both ligand and receptor in the first trimester deciduas from spontaneous abortions suggest, in physiological pregnancies, a possible interaction between CD30 and CD30-L by paracrine and/or autocrine pathways, as reported in malignant tumors (Trovato et al., 2001; Ruggeri et al., 2002), and in abortions, a probable distortion of these pathways that could even induce abortion.

In murine models, it has been demonstrated that the blockage of TNF receptor/ligand systems caus-

Table 4. Expression of CD30 and CD30-L in glandular and stromal cells of spontaneous abortion deciduas with inflammation signs.

\begin{tabular}{|c|c|c|c|c|}
\hline & $I^{\circ}$ trimester & & $I^{\circ}$ trimester & \\
\hline & CD30 & CD30-L & CD30 & CD30-L \\
\hline Glandular cells & $* 10( \pm 2.3)$ & $12( \pm 2.5)$ & $4.5( \pm 0.7)$ & $6( \pm 0.8)$ \\
\hline Stromal cells & $8( \pm 2.5)$ & $10( \pm 3.3)$ & $2( \pm 1.4)$ & $4.5( \pm 2.0)$ \\
\hline
\end{tabular}

${ }^{*}$ mean $( \pm S D)$ 
CD30

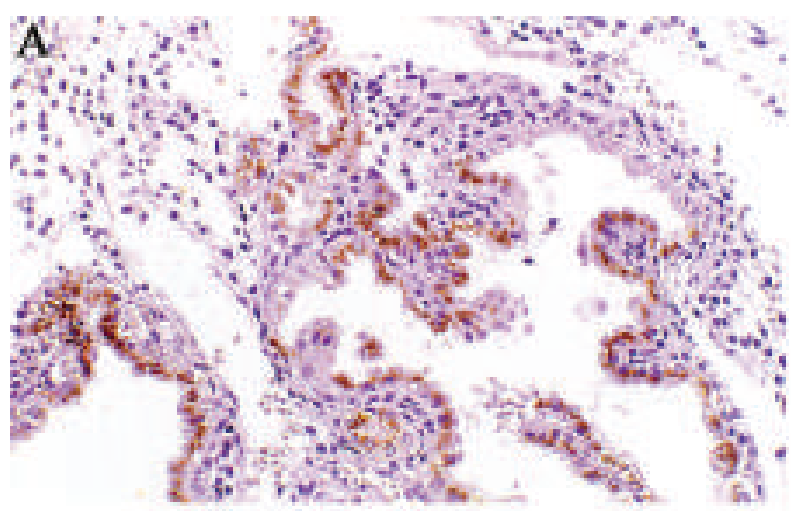

\section{CD30-Ligand}
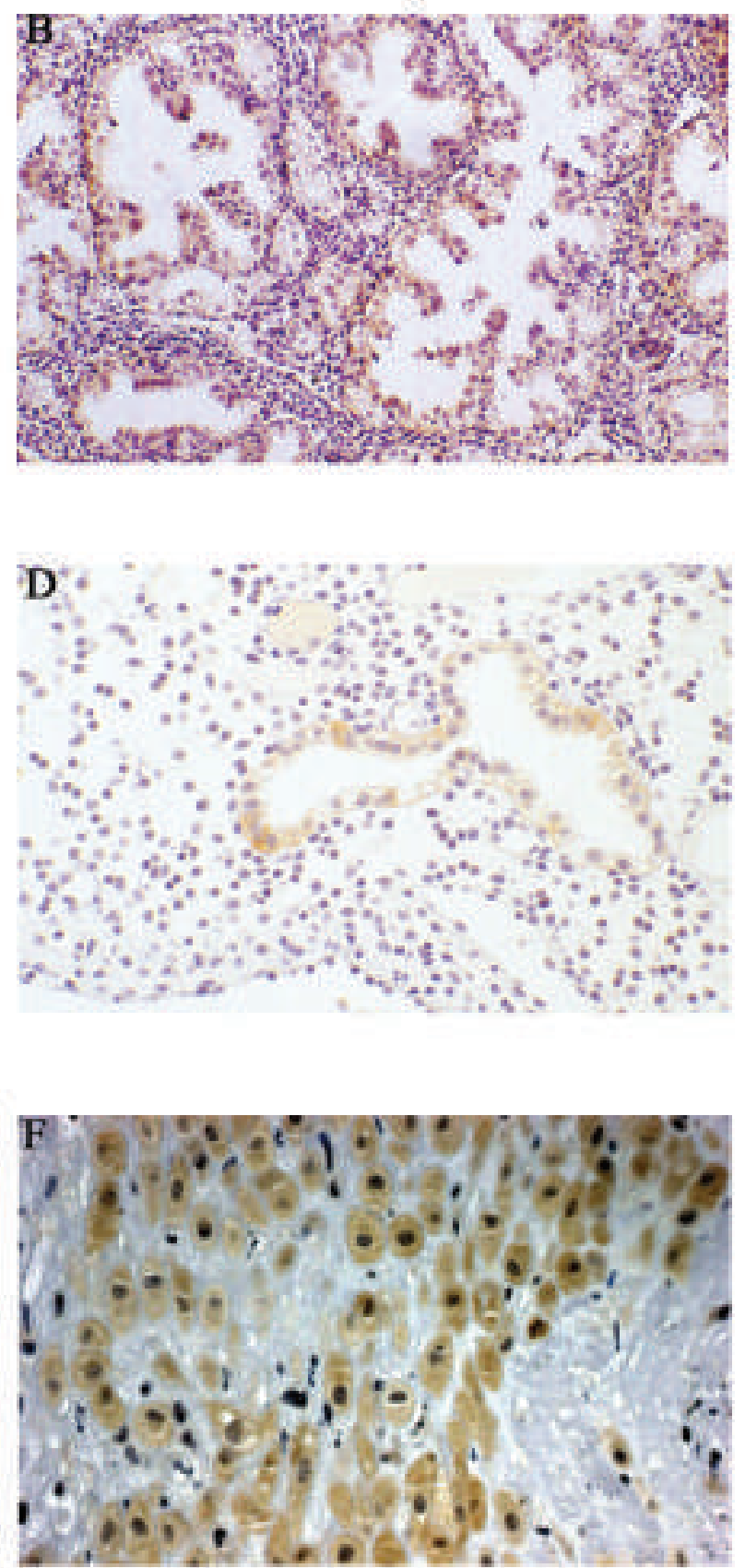

Figure 1. Intense CD30 (A) and moderate CD30-L (B) immunoreaction in glandular cells of first trimester physiological pregnancy deciduas (original magnification X130); intense CD30 (C) and weak CD30-L (D) immunostaining in glandular cells of first trimester spontaneous abortion deciduas without inflammation (original magnification X150 and X250, respectively); intense CD30 (E) and CD30-L (F) immunoreaction in stromal cells of first trimester spontaneous abortion deciduas without inflammation (original magnifications X300).

es early pregnancy loss (Erlebacher et al., 2004). In the present study, we found, in humans, an association between abortions and a reduced expression of CD30 and CD30-L confirming this murine evidence. In experimental models of miscarriages, it has been suggested that the TNF system may act by two different mechanisms; either by a systemic inhibition of ovarian function or by a local induction of inflammatory damage within trophoblast implantation sites (Erlebacher et al., 2004). The expression 
of the CD30/CD30-L system and its cellular pattern of expression detected in deciduas from spontaneous abortions with inflammation signs strongly suggest that this system, differently from CD40/CD40-L, acts at the maternal-fetal interface, possibly playing a role in the recruitment of inflammatory cells unreactive to CD30 or CD30-L. However, considering that CD30 was measured in the serum of pregnant woman (SverremarkEkström et al., 2001), it is correct not to exclude the possibility that systemic CD30 might directly interfere with the reproductive endocrine system or even trigger inflammatory mediators that influence the hormonal milieu.

\section{References}

Burns BF, Dardick I. Ki-1-positive non-Hodgkin's lymphomas. An immunophenotypic, ultrastructural, and morphometric study. Am J Clin Pathol 1990; 93, 327-32.

Carson DD, Bagchi I, Dey SK, et al. Embryo implantation. Dev Biol 2000; 223, 217-37.

Clark DA, Croitoru K.Th1/Th2, 3 imbalance due to cytokine-producing NK, gammadelta $T$ and $N K$-gammadelta $T$ cells in murine pregnancy deciduas in success or failure of pregnancy. Am $\mathrm{J}$ Reprod Immunol 2001; 45, 257-65.

Durkop H, Foss HD, Eitelbach F, et al. Expression of the CD30 antigen in non-lymphoid tissues and cells. J Pathol 2000; 190, 613-8.

Dürkop H, Latza U, Hummel M, Eitelbach F, Seed B, Stain H. Molecular cloning and expression of a new member of the nerve growth factor receptor family that is characteristic for Hodgkin's disease. Cell 1992; 68, 421-7.

Erlebacher A, Zhang D, Parlow AF, Glimcher LH. Ovarian insufficiency and early pregnancy loss induced by activation of the innate immune system. J Clin Invest 2004; 114, 39-48.

Falini B, Pileri S, Pizzolo G, et al. CD30 (Ki-1) molecule: a new cytokine receptor of the tumor necrosis factor receptor superfamily as a tool for diagnosis and immunotherapy. Blood 1995; 85, 1-14.

Fazleabas AT, Strakova Z. Endometrial function: cell specific changes in the uterine environment. Mol Cell Endocrinol 2002; 186, 143-7.

Ghosh D, Sengupta J. Recent developments in endocrinology and paraendocrinology of blastocyst implantation in the primate. Hum Reprod Updata 1998; 4, 153-68.

Gown AM, deWever N, Battifora H. Microwaved-based antigen unmasking. A revolutionary new technique for routeine immunohistochemistry. Appl Immunohistochem 1993; 1, 256-66.

Gruss HJ, Boiani N, Williams DE, Armitage RJ, Smith CA, Goodwin RG. Pleiotropic effects of the CD30 ligand on CD30-expressing cells and lymphoma cell lines. Blood 1994; 83, 2045-56.

Gruss HJ, Pinto A, Gloghini A, et al. CD30 ligand expression in non- malignant and Hodgkin's disease-involved lymphoid tissues. Am J Pathol 1996; 149, 469-81.

Ito K, Watanabe T, Horie R, Shiota M, Kawamura S, Mori S. High expression of the CD30 molecule in human decidual cells. Am J Pathol 1994; 145, 276-80.

Lee SY, Park CG, Choi Y. T cell receptor-dependent cell death of T cell hybridomas mediated by the CD30 cytoplasmic domain in association with tumor necrosis factor receptor-associated factors. J Exp Med 1996; 183, 669-674.

Locksley RM, Killeen N, Lenardo MJ. The TNF and TNF receptor superfamilies: integrating mammalian biology. Cell 2001; 104,487501.

Lockwood CJ, Nemerson Y, Guller S, et al. Progestational regulation of human endometrial stromal cells tissue factor expression during decidualizzation. J Clin Endocrinol Metab 1993; 76, 231-236.

Mechtersheimer G, Moller P. Expression of Ki-1 antigen (CD30) in mesenchymal tumors. Cancer 1990; 66, 1732-7.

Papadopoulos N, Galazios G, Anastasiadis P, et al. Human decidual cells can express the Hodgkin's cell-associated antigen Ki-l (CD30) in spontaneous abortions during the first trimester of gestation. Clin Exp Obstet Gynecol 2001; 28, 225-8.

Pellegrini P, Berghella AM, Contasta I, Adorno D. CD30 antigen: not a physiological marker for $\mathrm{TH} 2$ cells but an important costimulator molecule in the regulation of the balance between $\mathrm{TH} 1 / \mathrm{TH} 2$ response. Transpl Immunol 2003; 12, 49-61.

Phillips TA, Ni J, Hunt JS. Cell-specific expression of B lymphocyte (APRIL, BLyS)- and Th2 (CD30L/CD153)-promoting tumor necrosis factor superfamily ligands in human placentas. J Leukoc Biol 2003; 74, 81-7.

Raghupathy R, Makhseed M, Azizieh F, Hassan N, Al-Azemi M, AlShamali E. Maternal Th1- and Th2-type reactivity to placental antigens in normal human pregnancy and unexplained recurrent spontaneous abortions. Cell Immunol 1999; 196, 122-30.

Ruggeri RM, Villari D, Simone A, et al. Co-expression of interleukin-6 (IL-6) and interleukin-6 receptor (IL-6R) in thyroid nodules is associated with co-expression of CD30 ligand/CD30 receptor. J Endocrinol Invest 2002; 25, 959-66.

Saito S. Cytokine cross-talk between mother and the embryo/placenta. J Reprod Immunol 2001; 52, 15-33.

Salmon JE. A noninflammatory pathway for pregnancy loss: innate immune activation? J Clin Invest 2004; 114, 15-17.

Schwab U, Stein H., Gerdes J, et al. Production of a monoclonal antibody specific for Hodgkin and Sternberg-Reed cells of Hodgkin's disease and a subset of normal lymphoid cells. Nature 1982; 299, 65-7.

Smith CA, Gruss HJ, Davis T, et al. CD30 antigen, a marker for Hodgkin's lymphoma, is a receptor whose ligand defines an emerging family of cytokines with homology to TNF. Cell 1993; 73, 134960.

Sverremark Ekstrom ES., Bengtsson A, Svensson A, et al. Presence of CD30(+) and CD30L (+) cells in human placenta and soluble CD30 levels in cord blood are independent of maternal atopy. Placenta $2001 ; 22,372-9$

Trovato M, Villari D, Ruggeri RM, et al. Expression of CD30 ligand and CD30 receptor in normal thyroid and benign and malignant thyroid nodules. Thyroid $2001 ; 11,621-8$.

Wegmann TG, Lin H, Guilbert L, Mosmann TR. Bidirectional cytokine interactions in the maternal-fetal relationship : is successful pregnancy a TH2 phenomenon? Immunol Today 1993; 14, 353-6. 